\title{
BмJ Global Health Relation between opioid consumption and inclusion of opioids in 137 national essential medicines lists
}

\author{
Georgia C Richards, ${ }^{1}$ Jeffrey K Aronson, ${ }^{1}$ Carl Heneghan, ${ }^{1}$ Kamal R Mahtani, ${ }^{1}$ \\ Constantinos Koshiaris, ${ }^{2}$ Nav Persaud ${ }^{3,4}$
}

\begin{abstract}
To cite: Richards GC, Aronson JK, Heneghan C, et al. Relation between opioid consumption and inclusion of opioids in 137 national essential medicines lists. BMJ Global Health 2020;5:e003563. doi:10.1136/ bmjgh-2020-003563
\end{abstract}

Handling editor Eduardo Gómez

- Additional material is published online only. To view please visit the journal online (http://dx.doi.org/10.1136/ bmjgh-2020-003563).

Received 28 July 2020 Revised 9 September 2020 Accepted 6 October 2020

\section{Check for updates}

(c) Author(s) (or their employer(s)) 2020. Re-use permitted under CC BY-NC. No commercial re-use. See rights and permissions. Published by BMJ.

For numbered affiliations see end of article.

\section{Correspondence to} Georgia C Richards; georgia.richards@kellogg.ox. ac.uk

\section{ABSTRACT}

Introduction Opioids are deemed essential medicines by the World Health Organization (WHO). However, many countries have inadequate access to them. Whether including opioids in national essential medicines lists (EMLs) influences national opioid consumption has not been evaluated.

Methods We conducted a cross-sectional study to determine whether the listing of opioids in national EMLs was associated with consumption. We quantified the numbers and types of all opioids included in 137 national EMLs, for comparison with opioids in the WHO's Model List of Essential Medicines. Using the International Narcotics Control Board (INCB) consumption statistics for 2015-2017, we assessed the relation between annual mean opioid consumption (mg/person) and the numbers of opioids included in EMLs, controlling for region, population, healthcare expenditure, life expectancy, gross domestic product, human development and corruption.

Results Five opioids were included in the 20th edition of the WHO's Model List of Essential Medicines: codeine, fentanyl, loperamide, methadone and morphine. On average, countries' lists included significantly $(p<0.05)$ more opioids than the WHO's Model List. However, there were wide variations in the numbers (median 6 opioids; IQR: $5-9)$ and types $(n=33)$ of opioids included in national EMLs. Morphine (95\%), fentanyl (83\%) and codeine $(69 \%)$ were the most commonly included opioids. Most national EMLs were out of date (median publication date: 2011, IQR: 2009-2013). After adjusting for country characteristics, there was no relation between mean opioid consumption and the number of opioids in EMLs.

Conclusions Including opioids in national EMLs was not associated with consumption. National EMLs should be regularly updated to reflect the availability of opioids and the populations' needs for managing pain.

\section{INTRODUCTION}

Opioids are essential in managing pain, and other symptoms frequent in palliative care, opioid dependence and diarrhoeal diseases. ${ }^{1}$ Since the publication of its first list in 1977, WHO has included opioids in its Model List of Essential Medicines. ${ }^{2}$ Medicines selected by the WHO for inclusion in its Model List are of

\section{Key questions}

What is already known?

- WHO deems opioids as essential for managing pain palliative care, anaesthesia and opioid dependence.

- WHO encourages countries to adopt and adapt the WHO's Model List of Essential Medicines to identify national essential medicines lists (EMLs) to meet the priority health needs of their populations.

- Studies have not explored the relation between listing opioids in EMLs and consumption.

What are the new findings?

- National EMLs included a median of 6 (IQR: 5-9) opioids.

- Lists included significantly more opioids than the five opioids (ie, codeine, fentanyl, loperamide, methadone and morphine) in the WHO's Model List of Essential Medicines.

- Morphine, fentanyl, codeine, pethidine and tramado were the most commonly included opioids in lists.

- After adjusting for country characteristics, there was no relation between the number of opioids in EMLs and mean opioid consumption.

\section{What do the new findings imply?}

- Simply putting an opioid in an EML may not increase supply or change prescribing habits, which questions the usefulness of current EMLs in extending the accessibility of the most important medicines such as opioids.

- Revisions of current lists to reflect the availability of opioids and the populations' needs for managing pain, palliative care, anaesthesia and opioid dependence would be timely.

'utmost importance, and are basic, indispensable and necessary for the health needs of the population. ${ }^{2}$ WHO encourages governments to adopt and adapt the WHO's Model List to meet the needs of their populations.

Currently, 137 countries $(70 \%$ of 195 countries) serving more than 5 billion people have essential medicines lists (EMLs). ${ }^{3}$ Countries that implement the WHO's EMLs policies have improved the quality of usage 
of medicines. ${ }^{4}$ However, studies have highlighted considerable variation in the numbers and types of medicines included in EMLs. ${ }^{56}$ Researchers have evaluated the inclusion of medicines for neuropathic pain in national EMLs. ${ }^{6}$ However, this analysis only included four opioids (ie, tramadol, morphine, methadone and oxy-codone) and focused on low-income and middleincome countries. ${ }^{6}$ It is now recognised that adoption of a list of essential medicines could be beneficial in highincome countries, to reduce suboptimal prescribing and improve the affordability of essential medicines. ${ }^{78}$ However, the numbers and types of all opioids included by all countries with national EMLs have not been investigated. Nor is it known how the number of opioids included in a list is related to consumption.

The central aim of this study was to determine whether the listing of opioids in national EMLs is associated with consumption, as a proxy measure of accessibility, where consumption refers to the medical use of opioids and excludes recreational use. We also quantified the numbers and types of opioids in 137 national EMLs, for comparison with the WHO's Model List of Essential Medicines.

\section{METHODS}

\section{Study design and data sources}

We designed and conducted a cross-sectional observational study following the publication of the Global Essential Medicines (GEM) database ${ }^{9}$ and on receiving updated data from the International Narcotics Control Board (INCB $)^{10}$ in August 2019. The protocol for our study is openly available on the Open Science Framework (OSF; https://osf.io/385hx/). ${ }^{11}$

The International Narcotics Control Board (INCB), an independent body of the United Nations (UN), monitors implementation of international drug control conventions, including the Single Convention on Narcotic Drugs of 1961, which requires governments to report annual statistics on narcotic consumption relating to controlled drugs. ${ }^{12}$ Consumption refers to the total amount of a narcotic that is distributed for medical purposes at the retail level (ie, to institutions and programmes that are licensed to dispense to a patient). We received data from 2015 to $2017 \mathrm{in} \mathrm{kg}$ and removed 27 non-opioid substances (eg, cannabis, coca leaf and cocaine) to create a dataset of all opioids consumed. The included and excluded substances are listed in online supplemental box S1. We calculated a 3-year annual mean for each country with an EML. We adjusted for population size using 2016 data from the WHO Global Health Observatory ${ }^{13}$ to create a rate (ie, mean consumption in mg per person), see online supplemental box S2 for a sample calculation.

The GEM database was developed by Persaud $e t$ al in June 2017, by extracting all medicines listed by all countries with a national EML from the WHO's repository, as previously described, ${ }^{5}$ and all medicines listed in the 20th edition of the WHO's Model List of Essential Medicines ${ }^{1}$, which was the most up to date list published at the time the GEM database was created. In June 2019, WHO published the 21st edition of their Model List but there were no opioids added to the list, ${ }^{14}$ and thus, we used the 20th edition to be consistent with the GEM database. The medicines included in the database are coded using the Anatomical Therapeutic Classification (ATC) index. Two authors (GCR and JKA) independently searched the ATC index to create a list of opioids, compared their lists, discussed discrepancies and agreed on a master list of ATC codes for opioids; see online supplemental table $\mathrm{S} 1$. We used the ATC codes and medicine names in the master list to search for opioids in the GEM database for every country $(n=137)$ with an EML.

\section{Opioids included in EMLs}

We identified the numbers and types of opioids included in the 20th edition of the WHO's Model List of Essential Medicines, ${ }^{1}$ which was current when we began this study. We summed the number of opioids and identified the types of opioids for all countries with a national EML $\left(\mathrm{n}=137,70 \%\right.$ of 195 countries as defined by the $\left.\mathrm{UN}^{15}\right)$. We calculated the median and IQR for the number of opioids in EMLs. We calculated the percentage of opioids listed as a total of all included medicines, and compared countries' lists with the WHO's Model list using a onesample z-test and a significance level of 0.05. For each country, we calculated the numbers of opioids that were the same as or different from the WHO's Model List to create percentages of similarities and differences.

\section{Relation between opioid consumption and listing opioids in EMLs}

We extracted geographical region, population, healthcare expenditure per capita (US\$), life expectancy at birth (in years) for all sexes, gross domestic product (GDP) per capita, the human development index and the corruption perception scores for each country with an EML from web pages outlined in online supplemental table S2. The assumptions for untransformed linear regression were not met. Thus, we used a square root transformation of the dependent variable (ie, opioid consumption in $\mathrm{mg}$ /person), which improved the model. We conducted two multivariable analyses. In the first, we adjusted for GDP per capita and health expenditure per capita, as these variables had the least amount of missing data $(n=133)$. In the second analysis we adjusted for all country characteristics. We conducted a sensitivity analysis by removing extreme outliers.

\section{Statistical software and data access}

We used Stata V.16 ${ }^{16}$ for all statistical analyses and pandas and plotly modules in Jupyter Notebooks with Python v3 for choropleth maps. Our protocol, study materials, data and statistical code are all openly available on the OSF (https://osf.io/385hx/) ${ }^{11}$ and GitHub (https://github. com/georgiarichards/opioid_emls_maps). We used The Strengthening the Reporting of Observational Studies in 
Epidemiology (STROBE) reporting guidelines to write our manuscript; see the online supplemental 1 for the completed checklist.

\section{Protocol deviations}

We used INCB data from 2015 to 2017 instead of 2014 to 2016, as we obtained the most up-to-date data before we started the analysis (August 2019). We could not convert consumption from volume (ie, $\mathrm{kg}$ ) to morphine equivalents, because potency ratios are not available for all types of opioids included in our analysis. We did not conduct regression analyses for individual types of opioids as there were missing data; for example, only 73 countries (53\%) with EMLs reported consumption data for oxycodone; see online supplemental table S3.

\section{Patient and public involvement}

We involved three patients who have chronic pain and experience of taking opioids and other medicines for pain at the analysis phase of our research. Lead author (GCR) presented the preliminary findings to the patients during a formal face-to-face patient and public involvement meeting in December 2019. Patients provided suggestions for final analyses, the presentation of results, and the dissemination plans for our research. Preliminary findings were also presented to stakeholders at the inaugural Global Essential Medicines Meeting in November 2019 in Toronto, Canada, which included members of the WHO's Expert Committee on the Selection and Use of Essential Medicines. All stakeholders will be involved in the dissemination of our research.

\section{RESULTS}

Opioids listed in the WHO's Model List of Essential Medicines The 20th edition of the WHO's Model List of Essential Medicines included five opioids: codeine, fentanyl, loperamide, methadone and morphine; see online supplemental table S4. The included opioids account for $1.4 \%$ of all medicines listed in the WHO's Model List.

\section{Opioids listed in national EMLs}

EMLs included a median of six opioids (IQR: 5-9). Slovakia included the most opioids $(n=19)$ while Cambodia did not include any (see figure 1 and online supplemental table S5). There were 33 different opioids included in national EMLs (see figure 2 and online supplemental table S6). The most commonly included opioid was morphine (95\%), followed by fentanyl $(83 \%)$, codeine $(69 \%)$, pethidine $(65 \%)$ and tramadol $(62 \%)$ (see figure 2). The median publication date for EMLs was 2011 (IQR: 2009-2013; range: 2001-2017); (see online supplemental table S5).

\section{Comparison of national EMLs with the WHO's Model List}

Countries with EMLs included significantly more opioids $(\mathrm{z}=6.33, \mathrm{p}<0.05)$ as a percentage of all medicines than the WHO's Model List. Ninety-five per cent of countries included morphine, $83 \%$ listed fentanyl, $69 \%$ codeine, $61 \%$ loperamide and $41 \%$ methadone (see figure 3 ). Most countries $(98.5 \%)$ included at least one opioid recommended by the WHO, except for Cambodia, which listed no opioids, and Somalia which only listed pethidine. Eighteen per cent of countries (25 of 137) included all five opioids (ie, codeine, fentanyl, loperamide, methadone and morphine) included in WHO's Model list (see online supplemental figure S1). There were also a number of opioids included in national EMLs that were not included in the WHO Model Lists (see online supplemental figure S2).
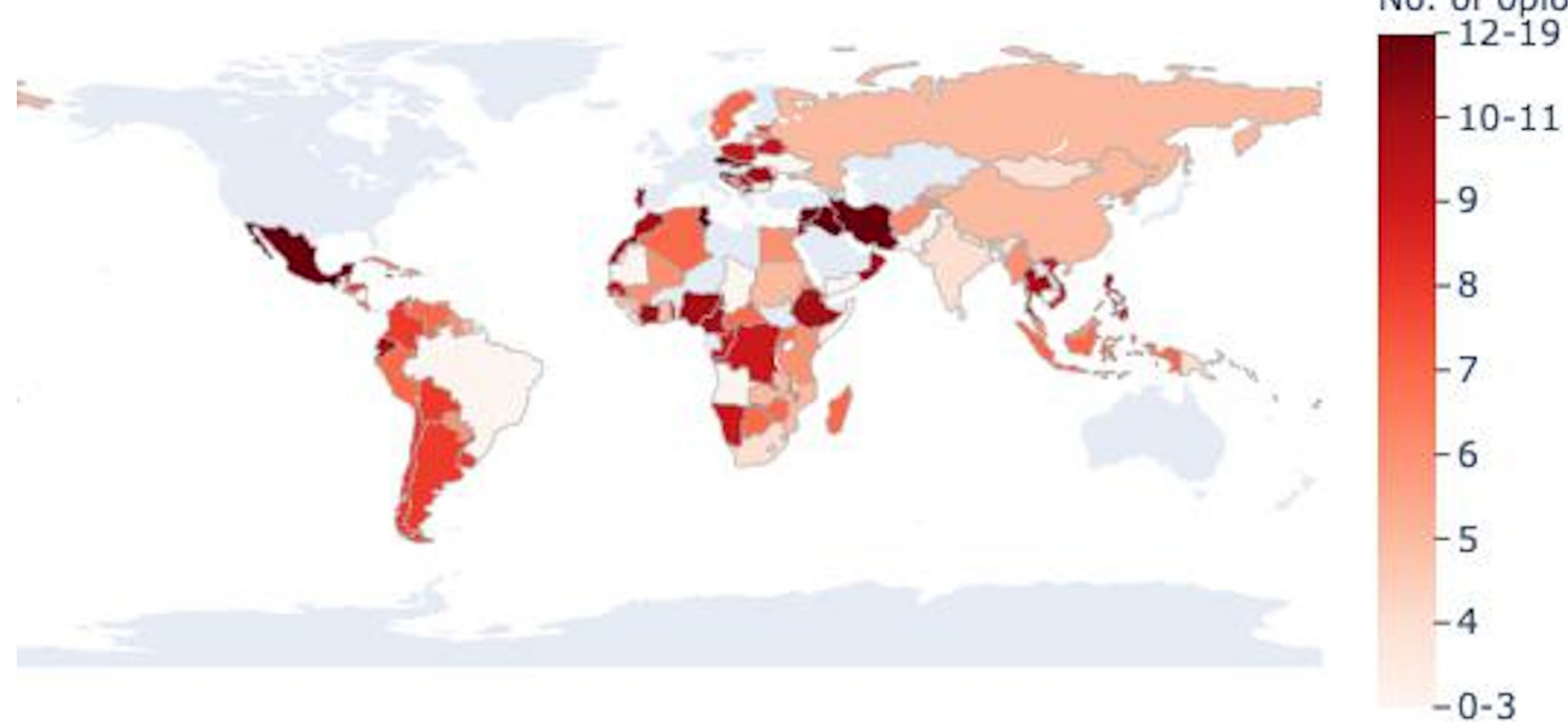

Figure 1 Number of opioids included in national essential medicines lists (EMLs) grouped by nine quantiles. There were 137 countries with EMLs; countries in light grey did not have an EML. 


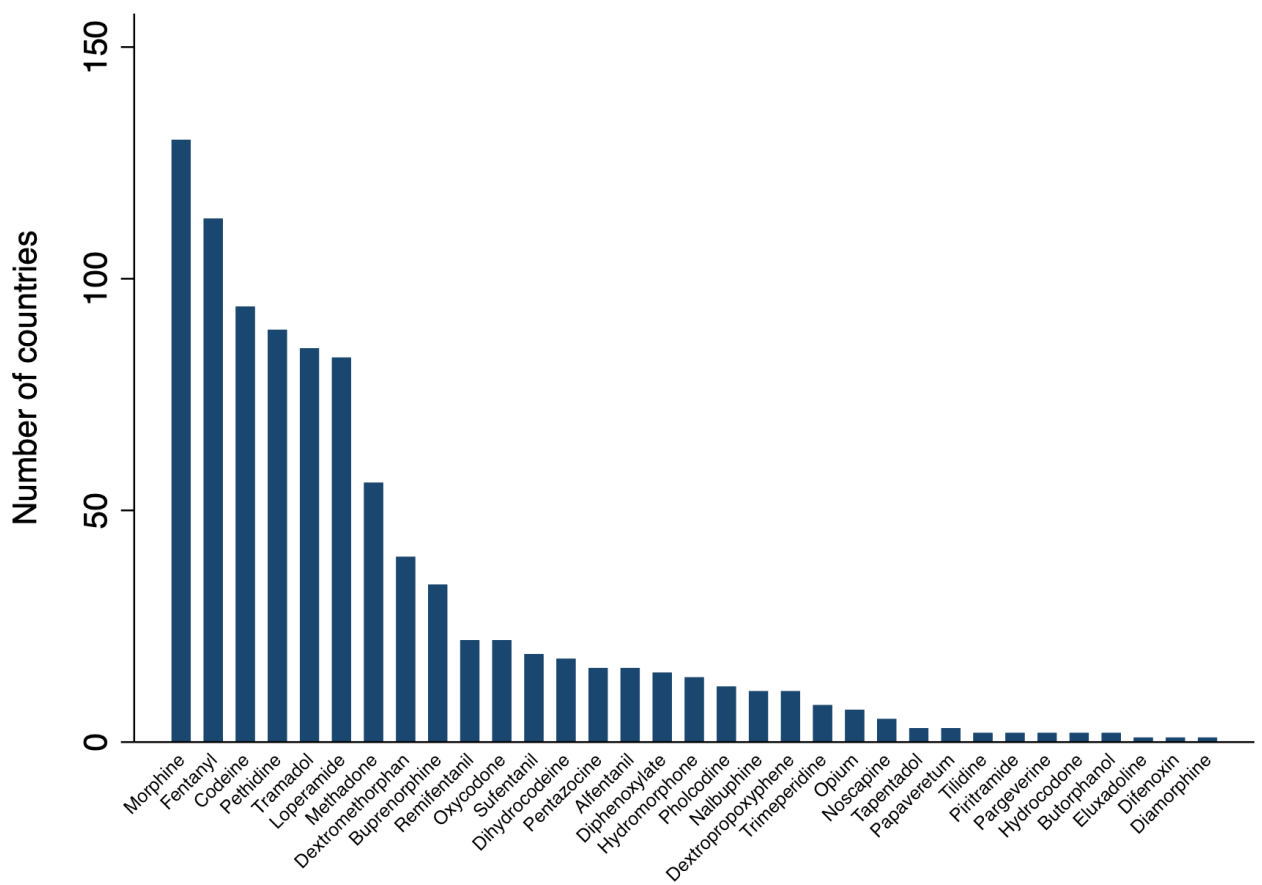

Figure 2 Types of opioids ( $n=33$ ) included in 137 national essential medicines lists ordered from most common to least.

\section{Relation between consumption and the number of opioids in EMLs}

Countries with EMLs consumed a median of $2 \mathrm{mg} /$ day (IQR: $0.13-6.8 \mathrm{mg} /$ day) of opioids between 2015 and 2017. There was a wide range in consumption (range: $0-97.9 \mathrm{mg}$ /day) with most countries $(93 \%, 128$ of 137$)$ consuming less than $20 \mathrm{mg} /$ person of opioids, and $17 \%$ of countries (23 of 137) reporting no opioid consumption. Countries that did not report consumption included a median of 5 opioids (IQR: 3-7; range: 0-12 opioids) in their national EML. In the univariable analysis, there was a positive and significant association between mean opioid consumption and the number of opioids listed in national EMLs (unadjusted coefficient: $0.172,95 \%$ CI 0.086 to $0.258, \mathrm{p}<0.0001$, table 1 ) but not after adjusting for GDP and healthcare expenditure (adjusted coefficient: $0.045,95 \%$ CI -0.022 to $0.111, p=0 \cdot 187$, table 1 ) or all country characteristics as summarised in online supplemental table S7 (coefficient: 0.0109, 95\% CI -0.0087 to $0.0305, \mathrm{p}=0.271$, table 1 ). In a sensitivity

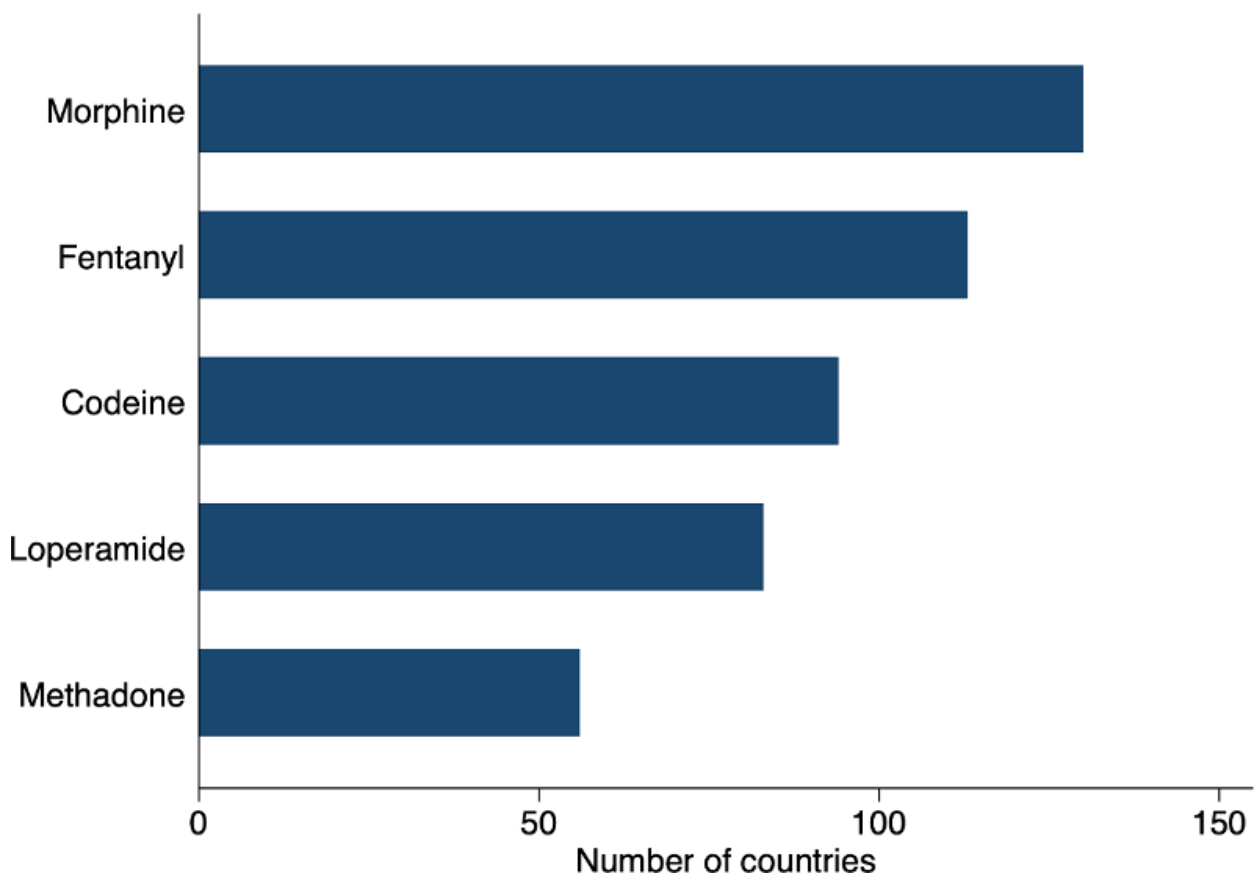

Figure 3 The five opioids in the WHO's Model List of Essential Medicines and the number of countries that included the five opioids in their national essential medicines lists. 


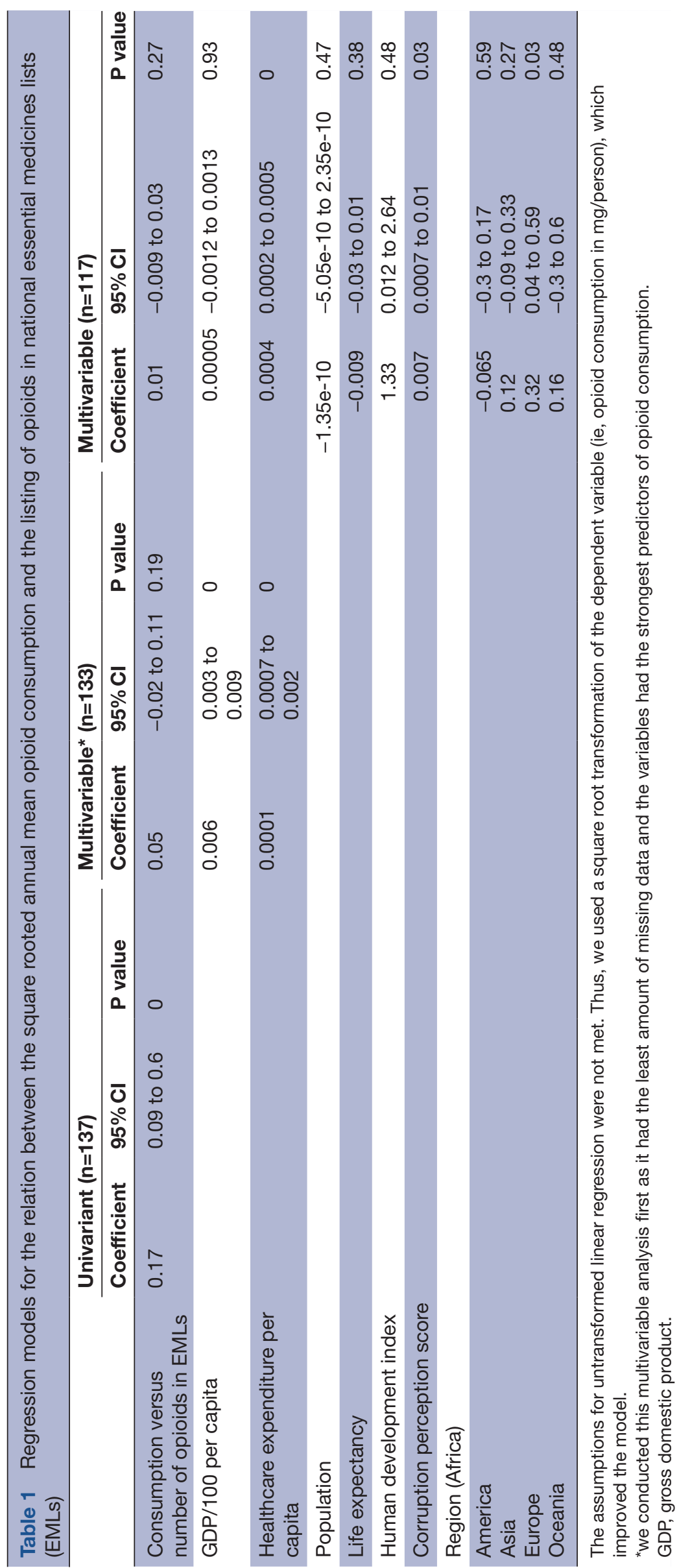




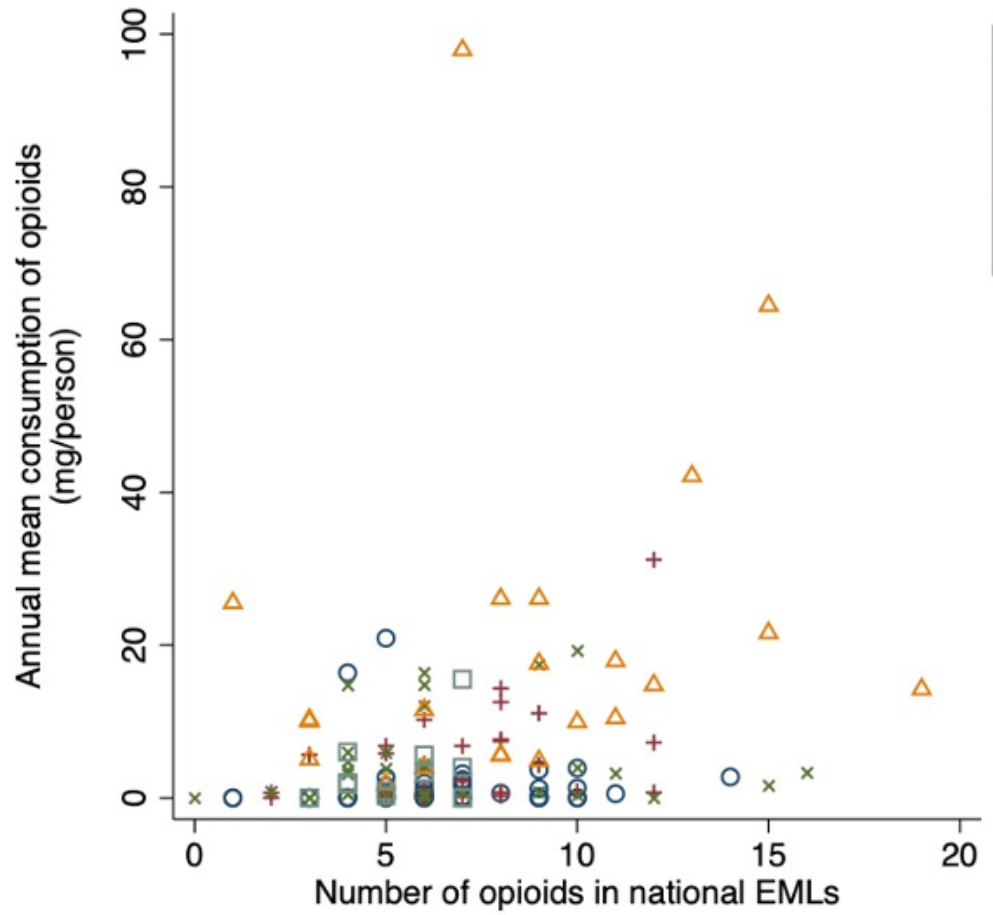

Figure 4 Scatter plot of the relation between annual mean opioid consumption (mg/person) for 2015-2017 and the number of opioids included in national essential medicines lists (EMLs) for 137 countries. Each country is represented by a symbol for its geographical region. After adjusting for country characteristics, there was no relation between consumption and the number of opioids in EMLs (coefficient: 0.0109, 95\% Cl: -0.0087 to $0.0305, p=0.271$ ).

analysis, removing the highest income country Sweden, the relation did not change. Figure 4 illustrates the relation between opioid consumption and the number of opioids included in EMLs before transformation; online supplemental figure S3 illustrates the relation after the transformation.

\section{DISCUSSION}

After adjusting for country characteristics, the number of opioids included in EMLs was not associated with the consumption of opioids. There are wide variations in the numbers and types of opioids included in national EMLs. Countries included significantly more opioids than the WHO Model List of Essential Medicines, whichincluded five opioids: morphine for anaesthesia; codeine, fentanyl, methadone and morphine for pain and palliative care; loperamide for diarrhoeal symptoms; and methadone for opioid use disorders. In national EMLs, morphine, fentanyl and codeine were the most common opioids included.

The purpose of EMLs, as originally stated by WHO in 1977, was to improve health, reduce costs of medicines, and extend the accessibility of the most necessary medicines to populations whose basic health needs could not be met by the existing supply system. ${ }^{2}$ The absence of a relation between the number of opioids in EMLs and consumption questions the usefulness of current EMLs in enabling access 'at all times, in adequate amounts, and in the proper dosage forms', as originally stated. ${ }^{2}$ However, most lists were out of date and most countries had low or no consumption of opioids. There may be other factors which influence this relation, such as cost implications imposed by EMLs, patchy implementation of lists, pharmaceutical interests that push more medicines into EMLs and restraints in the healthcare system on where and how medicines are prescribed, and by whom. ${ }^{17-21}$ However, these factors have not been explored in relation to opioids. Thus, future research should evaluate the function of EMLs in extending the accessibility of essential medicines like opioids in practice.

After the development of the first Model List of Essential Medicines, WHO encouraged countries to select medicines that meet the health priorities of their populations. Therefore, variations between countries is expected. However, including 19 different opioids, all with complex pharmacology, as found in Slovakia's EML, or not including any opioids as found in Cambodia's EML, may not adequately reflect differences in the health needs of those populations. Importantly, Cambodia has experienced recent outbreaks of HIV due to unsafe use of recreational opioids, ${ }^{22}$ and is a major transit route for exporting heroin, ${ }^{23}$ which may impact policies, access to pharmaceutical opioids, and health services in these regions. Future research could explore reasons for wide variations in the numbers and types of opioids included in national EMLs, and the reasons for adding or removing opioids in individual countries and regions.

Many advantages to using a central list of essential medicines have been identified. These include a reduction in the number of pharmaceutical products to be purchased, stored, analysed and distributed; an improvement in the quality of medicine utilisation, management, information and monitoring; stimulation of local 
pharmaceutical companies; assistance to low-income and middle-income countries in urgent need of high-priority medicine programmes to extend their primary healthcare provisions. ${ }^{24}$ WHO encourages countries to make the selection of essential medicines to be a continuing process that takes into account changing public health priorities, epidemiological conditions, progress in pharmacological and pharmacovigilance systems. ${ }^{2}$ We found that many lists were a number of years out of date based on the available information. Thus, revision of current EMLs to reflect such changes would be timely.

\section{Limitations}

The INCB and GEM databases do not specify formulations or dosage, and it is not possible to elucidate the clinical use of opioids consumed or included in EMLs. Thus, we recognise that medicines included in EMLs indicate nominal availability and thus caution is warranted when interpretating medicines on or absent from EMLs. Many high-income countries who consume most of the world's opioids $^{24}$ do not have EMLs, and therefore our regression model is not generalisable to all countries. Although our regression model was adjusted for a number of country characteristics, it is possible we may have missed some other important confounders. Consumption statistics are not reported to the INCB for opioids that are not regulated as internationally controlled substances (eg, tramadol and buprenorphine). Data reported to the INCB may also be late, unreported, or submitted inaccurately, as previously described. ${ }^{25}{ }^{26}$ The effects of different types of opioids vary by weight, which morphine equivalent conversion would account for, if accurate conversion were possible. We measured consumption using weight in mg adjusted for country population, as conversion factors for morphine equivalents, and defined daily doses (DDDs) are not available for all opioid substances included in our analysis. Thus, our findings may be less comparable to most previous research on opioid consumption that uses DDDs.

\section{CONCLUSIONS}

The number of opioids in lists was not associated with consumption, which questions the usefulness of current EMLs. The numbers and types of opioids included in 137 national EMLs differ from the WHO's Model List and vary between countries. Governments should consider updating their lists to reflect national availability of opioids and their population's needs for managing pain, symptoms frequent in palliative care, opioid dependence and diarrhoeal diseases.

\footnotetext{
Author affiliations

${ }^{1}$ Centre for Evidence-Based Medicine, Nuffield Department of Primary Care Health Sciences, University of Oxford, Oxford, UK

${ }^{2}$ Nuffield Department of Primary Care Health Sciences, University of Oxford, Oxford, UK

${ }^{3}$ Department of Family and Community Medicine, Faculty of Medicine, University of Toronto, Toronto, Ontario, Canada

${ }^{4}$ Centre for Urban Health Solutions, Department of Family and Community

Medicine, St Michael's Hospital, Toronto, Ontario, Canada
}

Twitter Georgia C Richards @Richards_G_C

Acknowledgements We thank the International Narcotic Control Board for sharing the raw narcotic consumption data. No direct funding was sought for this study. The lead and corresponding author (GCR) is financially supported by the NHS National Institute of Health Research (NIHR) School for Primary Care Research (SPCR), the Naji Foundation, and the Rotary Foundation, who were not involved in any aspect of the study. GCR had full access to all the data and had final responsibility for submitting the study for publication.

Contributors GCR devised the research question, designed the methods, wrote the protocol, conducted data screening, data cleaning and management, analysed the data, conducted the literature review and wrote the original manuscript. JKA conducted secondary data screening for the master list of opioids, reviewed preliminary findings, critically revised the manuscript and provided supervisory support. $\mathrm{CH}, \mathrm{KRM}$ and NP reviewed the protocol and preliminary findings, critically revised the manuscript and provided supervisory support. NP provided access to the Global Essential Medicines Database and was the catalyst for conducting this study. CK provided statistical advice, reviewed the statistical model and preliminary findings and critically revised the manuscript. All authors read, reviewed and approved the final manuscript.

Funding The lead author is financially supported by the National Institute for Health Research (NIHR) School for Primary Care Research (SPCR), the Naji Foundation and the Rotary Foundation.

Disclaimer The views expressed are those of the authors and not necessarily those of the NHS, the NIHR, or the UK Department of Health and Social Care.

Patient and public involvement statement We involved three patients who have chronic pain and experience of taking opioids and other medicines for pain at the analysis phase of our research. Lead author (GCR) presented the preliminary findings to the patients during a formal face-to-face PPIpatient and public involvement meeting in December 2019. Patients provided suggestions forn final analyses, the presentation of results, and the dissemination plans for our research. Preliminary findings were also presented to stakeholders at the inaugural Global Essential MedicinesGlobal Essential Medicines Meeting in November 2019 in Toronto, Canada, which included members of the WHO's Expert Committee on the Selection and Use of Essential Medicines. All stakeholders will be involved in the dissemination of our research.

Patient consent for publication Not required.

Ethics approval Not required

Provenance and peer review Not commissioned; externally peer reviewed.

Data availability statement Data are available in a public, open access repository. Our protocol, study materials, data and statistical code are all openly available on the Open Science Framework (https://osf.io/385hx/) and GitHub (https://github.com/georgiarichards/opioid_emls_maps).

Supplemental material This content has been supplied by the author(s). It has not been vetted by BMJ Publishing Group Limited (BMJ) and may not have been peer-reviewed. Any opinions or recommendations discussed are solely those of the author(s) and are not endorsed by BMJ. BMJ disclaims all liability and responsibility arising from any reliance placed on the content. Where the content includes any translated material, BMJ does not warrant the accuracy and reliability of the translations (including but not limited to local regulations, clinical guidelines, terminology, drug names and drug dosages), and is not responsible for any error and/or omissions arising from translation and adaptation or otherwise.

Open access This is an open access article distributed in accordance with the Creative Commons Attribution Non Commercial (CC BY-NC 4.0) license, which permits others to distribute, remix, adapt, build upon this work non-commercially, and license their derivative works on different terms, provided the original work is properly cited, appropriate credit is given, any changes made indicated, and the use is non-commercial. See: http://creativecommons.org/licenses/by-nc/4.0/.

\section{REFERENCES}

1 WHO. 20Th essential medicines list, 2017. Available: https://www. who.int/medicines/news/2017/20th_essential_med-list/en/ LB HW7p

2 WHO Expert Committee on the Selection of Essential Drugs \& World Health Organization. The selection of essential drugs : report of a WHO expert committee [meeting held in Geneva from 17 to 21 
October 1977], 1977. Available: https://apps.who.int/iris/handle/ $10665 / 41272$

3 Persaud N. Essential medicines and the challenges in the evidencebased manifesto. BMJ Evid Based Med 2018;23:123-4.

4 Holloway KA, Henry D. Who essential medicines policies and use in developing and transitional countries: an analysis of reported policy implementation and medicines use surveys. PLoS Med 2014;11:e1001724.

5 Persaud N, Jiang M, Shaikh R, et al. Comparison of essential medicines Lists in 137 countries. Bull World Health Organ 2019;97:394-404.

6 Kamerman PR, Wadley AL, Davis KD, et al. World Health organization essential medicines Lists: where are the drugs to treat neuropathic pain? Pain 2015;156:793-7.

7 Persaud N, Ahmad H. Canadian list of essential medications: potential and uncertainties. Can Fam Physician 2017;63:266-8.

8 Hogerzeil HV. The concept of essential medicines: lessons for rich countries. BMJ 2004;329:1169-72.

9 Persaud N, Jiang M, Shaikh R, et al. Global essential medicines database. Figshare 2019.

10 Thomas J. Availability of narcotic drugs for medical use. Int. Narc. Control Board 2019.

11 Richards GC, Aronson JK, Heneghan C, et al. Opioid consumption and the inclusion of opioids in 137 national essential medicines lists., 2020. Open Science FrameworkDOI: 10.17605/OSF.IO/385HX. Available: https://osf.io/385hx/[Accessed Sept 09, 2020]

12 INCB. Mandate-functions. Int. Narc. Control Board 2019.

13 WHO. Global health Observatory data Repository. World Heal. Stat 2019.

14 WHO. Who model Lists of essential medicines, 2019. Available: http://www.who.int/medicines/publications/essentialmedicines/en/ [Accessed 8 Sep 2020].

15 United Nations. Member states, 2019. Available: https://www.un.org/ en/member-states/ [Accessed 13 Feb 2020].

16 StataCorp. Stata statistical software 2019.
17 De Lima L, Pastrana T, Radbruch L, et al. Cross-Sectional pilot study to monitor the availability, dispensed prices, and affordability of opioids around the globe. J Pain Symptom Manage 2014;48:649-59.

18 Li DG, Najafzadeh M, Kesselheim AS, et al. Spending on world Health organization essential medicines in Medicare Part D, 201115: retrospective cost analysis. BMJ 2019;366:14257.

19 Hill AM, Barber MJ, Gotham D. Estimated costs of production and potential prices for the who essential medicines list. BMJ Glob Health 2018;3:e000571.

20 Faruqui N, Martiniuk A, Sharma A, et al. Evaluating access to essential medicines for treating childhood cancers: a medicines availability, price and affordability study in New Delhi, India. BMJ Glob Health 2019;4:e001379.

21 Droti B, O'Neill KP, Mathai M, et al. Poor availability of essential medicines for women and children threatens progress towards sustainable development goal 3 in Africa. BMJ Glob Health 2019;4:e001306.

22 Gokhale RH, Galang RR, Pitman JP, et al. A tale of 2 HIV outbreaks caused by unsafe injections in Cambodia and the United States, 2014-2015. Am J Infect Control 2017;45:106-7.

23 Chalk P. Southeast Asia and the Golden Triangle's Heroin Trade: Threat and Response. Studies in Conflict \& Terrorism 2000;23:89-106.

24 Berterame S, Erthal J, Thomas J, et al. Use of and barriers to access to opioid analgesics: a worldwide, regional, and national study. The Lancet 2016;387:1644-56.

25 Berterame S, Erthal J, Thomas J, et al. Use of and barriers to access to opioid analgesics: a worldwide, regional, and national study. Lancet 2016;387:1644-56.

26 Gilson AM, Maurer MA, Ryan KM, et al. Using a morphine equivalence metric to quantify opioid consumption: examining the capacity to provide effective treatment of debilitating pain at the global, regional, and country levels. J Pain Symptom Manage 2013;45:681-700. 